\title{
Using Josephson Vortex Lattices to Control Terahertz Radiation: Tunable Transparency and Terahertz Photonic Crystals
}

\author{
Sergey Savel'ev, ${ }^{1}$ A. L. Rakhmanov, ${ }^{1,2}$ and Franco Nori ${ }^{1,3}$ \\ ${ }^{1}$ Frontier Research System, The Institute of Physical and Chemical Research (RIKEN), Wako-shi, Saitama, 351-0198, Japan \\ ${ }^{2}$ Institute for Theoretical and Applied Electrodynamics, Russian Academy of Sciences, 125412 Moscow, Russia \\ ${ }^{3}$ Center for Theoretical Physics, Department of Physics, CSCS, University of Michigan, Ann Arbor, Michigan 48109-1120, USA
}

(Received 20 December 2004; published 22 April 2005)

The Josephson vortex (JV) lattice is a periodic array that scatters electromagnetic waves in the $\mathrm{THz}$-frequency range. We show that JV lattices can produce a photonic band-gap structure (THz photonic crystal) with easily tunable forbidden zones controlled by the in-plane magnetic field. The scattering of electromagnetic waves by JVs results in a strong magnetic-field dependence of the reflection and transparency. Fully transparent or fully reflected frequency windows can be conveniently tuned by the in-plane magnetic field. These proposals are potentially useful for controllable $\mathrm{THz}$ filters.

Layered superconducting structures, including strongly anisotropic (Bi-, Tl- and $\mathrm{Hg}$-based) high- $T_{c}$ superconductors (HTS) as well as artificial multilayered heterostructures $\left(\mathrm{Nb}-\mathrm{Al}-\mathrm{AlO}_{x}-\mathrm{Nb}\right)$ exhibit very intriguing physical properties. These layered media consist of superconducting and insulating layers which are parallel to the crystallographic $a b$ plane, and can be described as stacks of Josephson junctions (SJJ) [1]. When the external magnetic field $H_{a b}$ is applied parallel to the $a b$ plane, the Josephson vortices (JVs) penetrate the sample and form a triangular lattice. In contrast to Abrikosov or pancake vortices, the interaction between JV and crystal defects is weak and the $\mathrm{JV}$ lattice is near perfect at low enough temperatures. It was found [2-5] that the Josephson plasma frequency, $\omega_{J}$, of HTS layered systems is in the THz range, which is still hardly reachable for both electronic and optical devices. This is of particular interest for applications [6] and is fueling a sudden increase in studies of high-frequency properties of layered systems. The main focus so far has been on radiation produced by moving either a single $\mathrm{JV}$ or the entire JV lattice $[2,4,5]$ as well as studies of Josephson plasma waves in parallel magnetic fields $H_{a b}$ [7-9]. For instance, it was shown [10] how to control the THz radiation generated by fast moving JVs in spatially modulated samples of $\mathrm{Bi}_{2} \mathrm{Sr}_{2} \mathrm{CaCu}_{2} \mathrm{O}_{8+\delta}$. For out-of-plane modulated samples, the JVs moving in a subset of weaker junctions can generate out-of-ab-plane Cherenkov radiation [10]. For in-plane-modulated SJJ, moving JVs emit transition radiation [10] within certain frequency windows, which could be used for frequency-controlling $\mathrm{THz}$ emitters. In strong contrast to Ref. [10], here we focus on the scattering and filtering (not the emission) of $\mathrm{THz}$ electromagnetic waves (EMW) from a lattice of vortices which is fixed (as opposed to the moving vortices in [10]) inside a standard layered superconductor (in contrast to the artificially modulated ones in [10]).

By studying the influence of a fixed JV lattice on the propagation of $\mathrm{THz} \mathrm{EMW}$, we find that the interaction of the propagating wave and JV lattice results in forbidden gaps in the frequency spectrum (i.e., THz photonic crystal) conveniently tunable by the applied magnetic field $H_{a b}$. Moreover, by changing $H_{a b}$ one can easily change, by an order of magnitude, both the transmission, $T$, and reflection, $R=1-T$, coefficients of the EMW. Thus, the layered superconducting sample can operate as a THz-frequency filter tuned by the applied magnetic field $H_{a b}$. The manufacturing of artificial layered systems, with periodically modulated properties along the layers, would magnify the predicted effects.

Model. - We consider SJJ with layers in the $x z$ coordinate plane (Fig. 1 inset), which coincides with the $a b$ plane

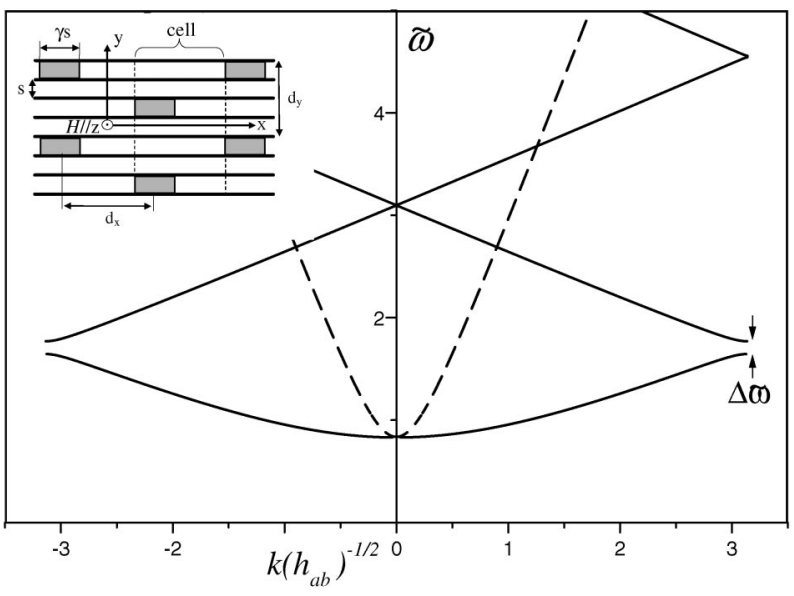

FIG. 1. Band-gap structure of an EMW propagating in a layered superconductor with a JV lattice: frequency $\tilde{\omega}$ of the EMW vs $k\left(h_{a b}\right)^{-1 / 2}$ with $k=x$-axis wave vector and the dimensionless magnetic field $h_{a b}=0.2$ and for $q s=0.3 \pi$ (solid line) and $q s=0.05 \pi$ (dashed line). The frequency gap (forbidden frequency range) between the first and the second zone is marked as $\Delta \tilde{\omega} \approx 0.2$. This gap diminishes for smaller $q(q=$ $y$-axis wave vector). Here, we use $s=15 \AA, \lambda_{a b}=2000 \AA$, $\gamma=600$. The inset shows the geometry of the problem. 
for HTS and the $y$ axis (along the $c$ axis) across the layers. The in-plane field $H_{a b}$ and, thus, the JVs are parallel to the $z$ axis. The distance $d_{x}$ between JVs in the lattice along the $x$ direction is much larger than along the $y$ direction, $d_{y}$, and $d_{x} / d_{y}=\gamma$. Here $\gamma$ is the anisotropy coefficient $(\gamma=$ 300-600 for HTS). The gauge-invariant phase difference $\varphi_{n}$ in SJJ can be described by the set of coupled sineGordon equations [11]

$$
\left(1-\frac{\lambda_{a b}^{2}}{s^{2}} \partial_{n}^{2}\right)\left(\frac{\partial^{2} \varphi_{n}}{\partial t^{2}}+\omega_{J}^{2} \sin \varphi_{n}\right)-\frac{c^{2}}{\varepsilon} \frac{\partial^{2} \varphi_{n}}{\partial x^{2}}=0 .
$$

Here $\lambda_{a b}$ is the London penetration depth across the layers, and $s$ is the interlayer distance $(10-20 \AA$ for HTS), the operator $\partial_{n}^{2} f_{n}=f_{n+1}+f_{n-1}-2 f_{n}, n$ is the layer number, $\varepsilon$ is the dielectric constant, $\omega_{J}=\sqrt{8 \pi e s J_{c} / \hbar \varepsilon}$ is the Josephson plasma frequency, and $J_{c}$ is the critical current density across the SJJ. We also neglect the relaxation term in Eq. (1), which is valid for samples thinner than the skin depth ( $\sim 0.3 \mathrm{~mm}$ for Bi2212). For larger samples absorption of EMW has to be taken into account. However, the picture discussed below remains qualitatively correct, because the characteristic scale of the EMW decay is still much larger than the distance between JVs for the magnetic fields considered.

We consider the EMW $\mathbf{H}(x, y, t)=\hat{\mathbf{z}} H_{0}(x) \times$ $\exp (i q y-i \omega t)$, propagating through the sample, where $\hat{\mathbf{z}}$ is the unit vector along $z$ (i.e., EMW with the magnetic field along the direction of the JVs). We assume that the amplitude $H_{0}$ is small compared to $H_{a b}$ and the solution to Eq. (1) can be obtained perturbatively as $\varphi_{n}=\varphi_{n}^{0}+\varphi_{n}^{1}$, where $\varphi_{n}^{0}$ corresponds to the steady JV lattice and $\varphi_{n}^{1}$ is related to propagating waves, $\left|\varphi_{n}^{0}\right| \gg\left|\varphi_{n}^{1}\right|$. For moderate magnetic fields, the steady-state solution can be approximated as a sum, $\varphi_{n}^{0}=\sum_{m} \varphi^{0}\left(x-x_{m n}\right)$, of solitons [12] $\varphi_{0}=\pi+2 \tan ^{-1}\left(x / l_{0}\right)$, where $2 l_{0}=\gamma s$. Here $x_{m n}$ is the position of the $m$ th JV in the $n$th layer. Below we assume that the JV lattice is fixed. Note that the JV lattice can be easily pinned by pancake vortices generated by a low outof-plane magnetic field (see, e.g., [13]).

Substituting $\quad \varphi^{1}(x, y, t)=\psi(x) \exp (i q y-i \omega t) \quad$ into Eq. (1) and averaging over $y$ for $|q|<\pi / s$, we derive in the linear approximation

$$
\psi^{\prime \prime}(\eta)-\kappa_{0}^{2}(q)\left[\tilde{\omega}_{J}^{2}(\eta)-\tilde{\omega}^{2}\right] \psi(\eta)=0,
$$

where the following dimensionless variables were introduced: $\eta=x / \gamma s, \tilde{\omega}=\omega / \omega_{J}, \tilde{\omega}_{J}(\eta)=\omega_{J}(\eta) / \omega_{J}$,

$$
h_{a b}=\frac{\gamma s^{2} H_{a b}}{2 \phi_{0}}, \quad \kappa_{0}^{2}(q)=\left(\frac{s}{\lambda_{a b}}\right)^{2}\left(1+q^{2} \lambda_{a b}^{2}\right),
$$

where $\phi_{0}$ is the flux quantum. When deriving Eqs. (2) and (3), we use the relations $\lambda_{c}=c / \sqrt{\varepsilon} \omega_{J}$ and $\lambda_{c} / \lambda_{a b}=\gamma$. The function $\tilde{\omega}_{J}^{2}(\eta)$ :

$$
\tilde{\omega}_{J}^{2}(\eta)=\left\langle\sum_{m} \cos \left(\varphi_{n m}^{0}\right)\right\rangle_{n},
$$

has a period $d_{x}$ along the $x$ direction, where $\langle\ldots\rangle_{n}$ denotes an average over the layers [14]. The physical meaning of the modulation $\tilde{\omega}_{J}(\eta)$ of the Josephson frequency is that the effective critical current of the layered medium becomes modulated due to the current suppression near the JV cores.

Equation (2) is an ordinary linear differential equation with a periodic coefficient. It can be solved numerically, or approximately by the WKB method. Alternatively, this equation has a Schrödinger equation form with a "potential" $\tilde{\omega}_{J}^{2}(\eta)$. For qualitative studies and estimates, one can approximate the dependence $\tilde{\omega}_{J}^{2}(\eta)$ by an appropriate stepwise function:

$$
\tilde{\omega}_{J}^{2}(\eta)=1-\frac{3 \sqrt{h_{a b}}}{2} \sum_{m} F\left(\eta-\frac{m}{\sqrt{h_{a b}}}\right),
$$

where $F(\eta)=1$ if $|\eta|<1$ and $F=0$ if $|\eta|>1$ and we use the relation $2 \phi_{0} /\left(d_{x} d_{y}\right)=H_{a b}$. When deriving Eq. (5) we assume, as usual, that the core of each JV is $\gamma s$ along the $x$ direction and $s$ (i.e., one layer) along the $y$ - direction. Outside the cores $\cos \left(\varphi_{m n}^{0}\right)=1$, while inside the cores (shadowed regions in Fig. 1 inset) $\cos \left(\varphi_{m n}^{0}\right)=-1 / 2$. The second order equation (2) requires the continuity of both $\psi(\eta)$ and $\psi^{\prime}(\eta)$ in the sample.

Band-gap structure. -Forbidden zones in the $\omega(k)$ dependence, or so-called "photonic crystal" [15], can occur when the EMW propagates through a periodically modulated structure, e.g., through the JV lattice. The dimensionless spatial period of the structure is $1 / \sqrt{h_{a b}}$. Following the usual band-theory approach, we obtain the solution of Eq. (2) in the form of the Bloch wave $\psi(x)=u(x, k) \times$ $\exp (i k x)$, where $u(x, k)$ is a periodic function with the period $1 / \sqrt{h_{a b}}$ and the dimensionless wave vector $k$ is in the first Brillouin zone, $-\pi \sqrt{h_{a b}}<k<\pi \sqrt{h_{a b}}$. The solution of the linear Eq. (2) within one elementary cell $j$ (inset in Fig. 1) is a sum of exponential terms multiplied by constants $C_{j}$. Using the continuity of $\psi$ and $\psi^{\prime}$ at the discontinuities of $\tilde{\omega}(\eta)$, and the periodicity of these functions, we obtain a set of homogeneous linear equations for $C_{j}$. The nontrivial solution of these equations exists only if the determinant of the set of equations is zero. Then, after straightforward algebra, we obtain the dispersion equation for $\omega(k)$ in the form

$$
\begin{aligned}
& \cos \left(\kappa_{1} b\right) \cos \left(\kappa_{2}\right)-\frac{\kappa_{1}^{2}+\kappa_{2}^{2}}{2 \kappa_{1} \kappa_{2}} \sin \left(\kappa_{1} b\right) \sin \left(\kappa_{2}\right) \\
& =\cos [k(b+1)],
\end{aligned}
$$

where $b=1 / \sqrt{h_{a b}}-1$ and

$$
\kappa_{1}=\kappa_{0}\left(\tilde{\omega}^{2}-1\right)^{1 / 2}, \quad \kappa_{2}=\kappa_{0}\left(\tilde{\omega}^{2}+\frac{3}{2} \sqrt{h_{a b}}-1\right)^{1 / 2} .
$$


In Fig. 1 the dependence $\tilde{\omega}(k)$ is shown for two different wave vectors $q$. The ratio $s / \lambda_{a b}$ is small and the most pronounced effect occurs at high $q$ when $q \gg \lambda_{a b}$ and $\kappa_{0}=q s$. The value of $\Delta \tilde{\omega}$ is gradually suppressed when decreasing $H_{a b}$ and decays fast when $q$ decreases or the frequency increases.

Tunable transparency. - The THz photonic crystal discussed above indicates that the JV lattice can significantly affect the transparency of the medium. Here we calculate the transmission and reflection coefficients for the EMW emitted by some internal source [inset in Fig. 2(a)], e.g., by the moving JVs. The solution of Eq. (2) for the $j$ th cell of the magnetic structure can be expressed in the vector form $\vec{\psi}_{\alpha}^{j}=\left\{C_{1 \alpha}^{j} \exp \left(i \kappa_{\alpha} x\right) ; C_{2 \alpha}^{j} \exp \left(-i \kappa_{\alpha} x\right)\right\}$, where $\alpha=1,2$ and $C_{i \alpha}^{j}$ are constants. This is not a periodic solution and we need to impose the continuity of $\psi$ and $\psi^{\prime}$ at any discontinuity of the function $\tilde{\omega}^{2}(\eta)$. As a result, we obtain a set of linear equations relating $\vec{\psi}_{\alpha}^{j-1}$ and $\vec{\psi}_{\alpha}^{j}$. The solution of these equations can be presented in a symbolic form $\vec{\psi}_{\alpha}^{j}=\hat{L} \vec{\psi}_{\alpha}^{j-1}$, where $\hat{L}$ is a $2 \times 2$ matrix. Then, we use a linear nondegenerate transformation $\hat{G}$ that diagonalizes $\hat{L}$.
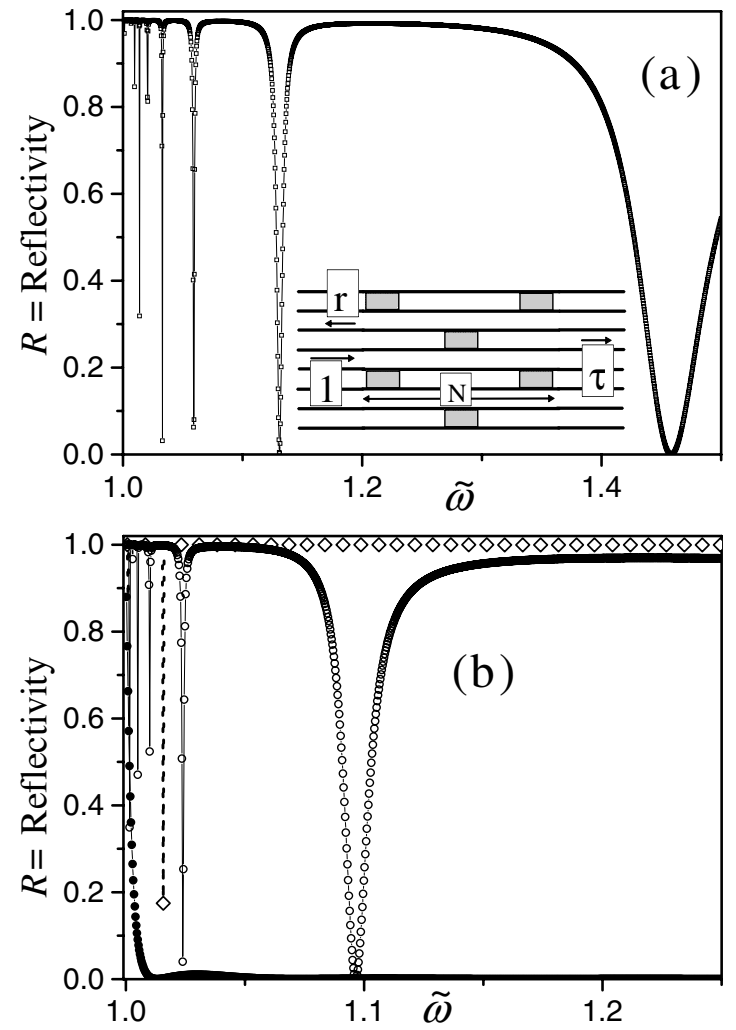

FIG. 2. Internal reflection: EMWs emitted (by a moving JV) inside a sample (inset) reflect back with intensity $R=|r|^{2}$ and transmit with intensity $T=|\tau|^{2}=1-R$. The reflection coefficient $R$ vs the EMW frequency $\tilde{\omega}$ for a sample with length $l=$ $100 \gamma s$; (a) for $h_{a b}=0.2, q s=0.3 \pi$, and (b) for $q s=0.05 \pi$, $h_{a b}=0.2$ (diamond), $h_{a b}=0.05$ (open circles), $h_{a b}=0$ (solid circles). Other parameters are the same as in Fig. 1.
By applying $N$ times such a procedure, we find the linear transformation

$$
\vec{\psi}_{\alpha}^{N}=\hat{G}^{-1}\left(\hat{G} \hat{L} \hat{G}^{-1}\right)^{N} \hat{G} \vec{\psi}_{\alpha}^{0}
$$

that propagates the solution from the zeroth to $N$ th elementary cell.

Here we only consider the case of frequencies higher than the plasma frequency. We denote the amplitude of the incident wave $C_{11}^{0}$ as 1 , the amplitude of the reflected wave $C_{12}^{0}=r$, and the amplitude of the transmitted wave $C_{11}^{N}$ as $\tau$ [Fig. 2(a) inset]. Using Eq. (7) we obtain two linear equations for two independent variables, $r$ and $\tau$, since $C_{12}^{N}=0$. By solving these equations, we find

$$
r=\beta_{2} \frac{M_{2}^{N}-M_{1}^{N}}{M_{1}^{N}-\beta_{2} \beta_{1} M_{2}^{N}},
$$

where

$$
\begin{aligned}
& M_{1,2}=\left[\cos \kappa_{2}(b-1) \pm i \frac{\kappa_{1}^{2}+\kappa_{2}^{2}}{2 \kappa_{1} \kappa_{2}} \sin \kappa_{2}(b-1)\right] e^{\mp i(b-1) \kappa_{1}} \\
& \beta_{1,2}= \pm \frac{M_{2}-M_{1}+\sqrt{\left(M_{2}-M_{1}\right)^{2}+4 L_{1} L_{2}}}{2 L_{1,2}} \\
& L_{1,2}= \pm i \frac{\kappa_{1}^{2}-\kappa_{2}^{2}}{2 \kappa_{1} \kappa_{2}} \sin \kappa_{2}(b-1) e^{ \pm i(b-1) \kappa_{1}}
\end{aligned}
$$

The frequency dependence of the reflection coefficient $R=|r|^{2}$ is shown in Fig. 2 for different magnetic fields $H_{a b}$ and $y$-axis wave vectors $q$. The transparency (transmission $T$ ) of the crystal increases when increasing the frequency $\tilde{\omega}_{J}$ and when decreasing either the sample length $l=\gamma s N / \sqrt{h_{a b}}$ or $H_{a b}$, due to the decrease of the number of scattering layers. The frequency dependence of the reflection $R$ or transmission $T$ coefficients is much more interesting for large $q$, when the interaction of the EMW and the JVs becomes stronger. In this case, the oscillations in the frequency dependence of $R(\omega)$ and $T(\omega)=|\tau|^{2}$ are obtained due to the interference of the transmitting and reflecting waves[16]. Moreover, close to the forbidden frequency zones, the dependence of $R$ and $T$ versus $\omega$ has several characteristic deep and narrow peaks. At $q=0$ the corresponding functions are monotonous. Varying the applied magnetic field $H_{a b}$ tunes the reflection at a given frequency from 0 to 1 . In a long sample this tuning remains significant even at small $q$, due to cumulative effect of a large number of weak scatterers.

Now we consider an incident wave propagating from the vacuum through the sample along the $a b$ planes. In this case the value of $q s$ is small for THz-range radiation since in vacuum $k^{2}+q^{2}=\omega^{2} / c^{2}$, while $s$ is in the nanometer range. For $\omega / 2 \pi=1 \mathrm{THz}$ and $s=2 \mathrm{~nm}$ we find the estimate $q s \leq 4.19 \times 10^{-5}$. Assuming below $q=0$ and using the well-known relations between the phase $\varphi$ and electromagnetic field, one can find $H=-\phi_{0} \varphi^{\prime}(x) / 2 \pi s$, and $E_{y}=i \omega \phi_{0} \varphi(x) / 2 \pi s c$. Then imposing the continuity 

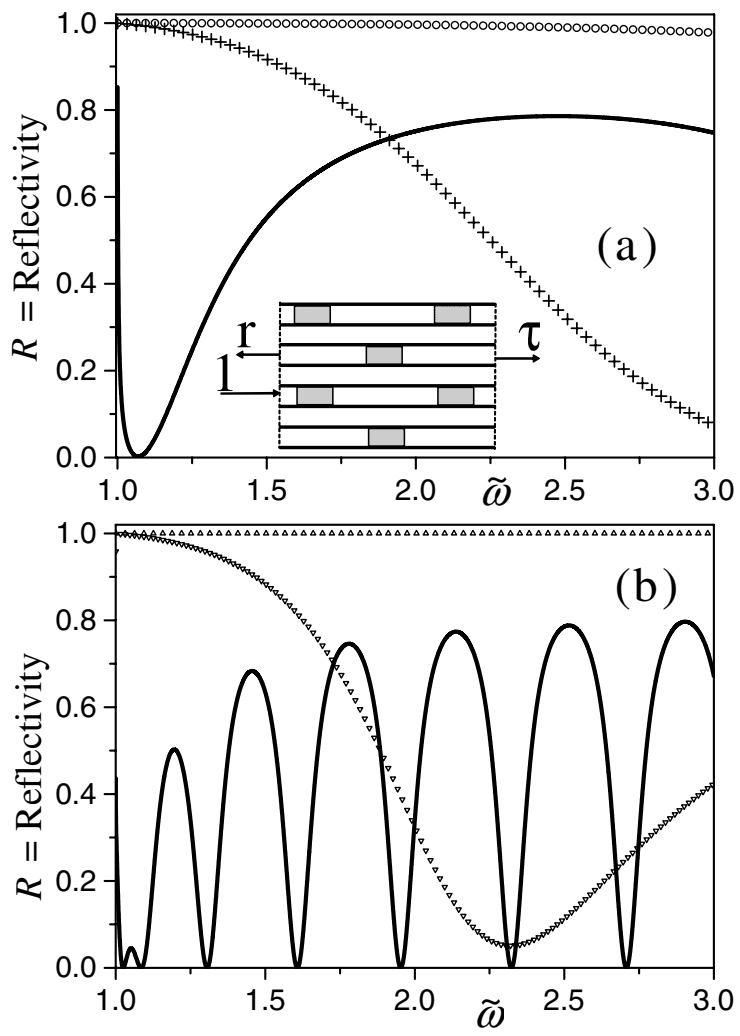

FIG. 3. Reflection of electromagnetic waves from the sample, or THz "filter" shown in the inset: the reflection coefficient $R$ vs $\tilde{\omega}$ for an EMW propagating from the vacuum at $q=0$, (a) for $l=100 \gamma s, h_{a b}=0.1$ (open circles), $h_{a b}=0.01$ (crosses), $h_{a b}=0$ (solid line); and (b) for $l=1000 \gamma s, h_{a b}=0.1$ (up triangles), $h_{a b}=0.001$ (down triangles), $h_{a b}=0$ (solid line). Other parameters are the same as in Fig. 1. The deep minima for the solid line in (b) is due to the reflection at the sample boundaries.

of both $H$ and $E_{y}$ at the sample surface and using Eq. (7), we find the expression for the amplitude $r$ of the reflected wave

$$
\begin{aligned}
r & =\frac{1+Z(\tilde{\omega}) D(\tilde{\omega}) \exp \left(-2 \kappa_{1} b\right)}{Z(\tilde{\omega})+D(\tilde{\omega}) \exp \left(-2 \kappa_{1} b\right)}, \\
D & =\frac{\beta_{1}\left(Z+\beta_{2}\right) M_{2}^{N}-\left(1+\beta_{1} Z\right) M_{1}^{N}}{\left(Z+\beta_{2}\right) M_{2}^{N}-\beta_{2}\left(1+\beta_{1} Z\right) M_{1}^{N}},
\end{aligned}
$$

where $Z=\left(\kappa_{1}-\tilde{\omega} g\right) /\left(\kappa_{1}+\tilde{\omega} g\right)$ and $g=s /\left(\sqrt{\varepsilon} \lambda_{a b}\right)$. We assume that two flux-free zones with thickness $b$ exist near the sample edges. Note that Eq. (8) corresponds to $Z=0$ in Eq. (10).

The calculated frequency dependence of the reflection coefficient is shown in Fig. 3 for $q=0$ at different magnetic fields and different sample lengths. The transparency increases with the increase of the frequency and with the decrease of the number of scattering layers due to a decrease of the magnetic field $H_{a b}$ or due to a decrease of the sample length. The oscillation in the transition and reflection coefficients occurs due to the interference of the scattered and transmitted waves on JVs and sample boundaries. These frequency windows can be easily tuned by the in-plane magnetic field $H_{a b}$.

Conclusions. - The reflection and transmission of EMW in a layered superconducting medium is affected by the presence of Josephson vortices, either in anisotropic high- $T_{c}$ or artificial superconductors. The interaction of the EMW with the array of Josephson vortices gives rise to a tunable $\mathrm{THz}$ photonic crystal and a rather peculiar frequency dependence of the transmission and reflection coefficients. Thus, the propagating EMW can be tuned by varying the in-plane magnetic field $H_{a b}$. The same effects are expected for the artificial structures with periodically modulated properties. However, in this case the properties of the system would be not so easily tunable. In general, the transmitted wave should be partly polarized since only waves with magnetic field along the $a b$ plane can propagate through the layered system. These features are potentially useful for $\mathrm{THz}$ filters.

We gratefully acknowledge conversations with Professor M. Tachiki and partial support from the NSA and ARDA under AFOSR Contract No. F49620-02-10334, and by the NSF Grant No. EIA-0130383.

[1] G. Blatter et al., Rev. Mod. Phys. 66, 1125 (1994).

[2] J. Zitzmann et al., Phys. Rev. B 66, 064527 (2002).

[3] Y. Tominari et al., Appl. Phys. Lett. 80, 3147 (2002).

[4] M. Tachiki et al., Phys. Rev. B (to be published).

[5] G. Hechtfischer et al., Phys. Rev. Lett. 79, 1365 (1997).

[6] See, e.g., the special issue of Philos. Trans. 362, No. 1815 (2004).

[7] I. Kakeya et al., Physica C (Amsterdam) 378-381, 437 (2002).

[8] T. Koyama, Phys. Rev. B 68, 224505 (2003); H. Matsumoto and T. Koyama, Physica C (Amsterdam) 412-414, 444 (2004).

[9] Y. Matsuda et al., Phys. Rev. B 55, R8685 (1997).

[10] S. Savel'ev, V. A. Yampolskii, A. L. Rakhmanov, and F. Nori (to be published).

[11] S. N. Artemenko and S. V. Remizov, Physica C (Amsterdam) 362, 200 (2001).

[12] A. Gurevich, Phys. Rev. B 46, R3187 (1992).

[13] A.E. Koshelev, Phys. Rev. Lett. 83, 187 (1999); S. Savel'ev and F. Nori, Nat. Mater. 1, 179 (2002).

[14] This averaging is valid for $q s<\pi \sqrt{h_{a b}}$ or at any $q$ for $h_{a b}=0$.

[15] E. Yablonovitch, Phys. Rev. Lett. 58, 2059 (1987); J. D. Joannopoulos, R.D. Meade, and J.N. Winn, Photonic Crystals (Princeton University, Princeton, NJ, 1995); R. E. Slusher and B.J. Eggleton, Nonlinear Photonic Crystals (Springer, New York, 2003); K. Sakoda, Optical Properties of Photonic Crystals (Springer, New York, 2001).

[16] L. D. Landau, E. M. Lifshitz, and L.P. Pitaevskii, Electrodynamics of Continuous Media, (ButterworthHeinemann, Oxford, 1995). 